\title{
A Three-phase Large Scale Skyline Service Selection Framework in Clouds
}

\author{
Jinzhong $\mathrm{LI}^{1,2,3}$, Jintao ZENG ${ }^{1,2,{ }^{4}}$, Lei PENG ${ }^{1,2}$, Wenlang Luo ${ }^{1,2 *}$ \\ ${ }^{1}$ Department of Computer Science and Technology, \\ Jinggangshan University, Ji'an Jiangxi 343009, China \\ ${ }^{2}$ Key laboratory of watershed ecology and geographical environment \\ monitoring, NASG, Jinggangshan University, Ji'an Jiangxi 343009, China \\ ${ }^{3}$ Department of Computer Science and Technology, \\ Tongji University, Shanghai 201804, China \\ ${ }^{4}$ Department of Surveying and Geo-Informatics, Tongji University, \\ Shanghai 200092, China \\ 1210510@tongji.edu.cn,wen8102011@126.com
}

\begin{abstract}
For the large scale services with high-dimensional QoS attributes and distributed environment, traditional service selection approaches are faced with unprecedented challenges in terms of efficiency and performance of QoS. To address these challenges, we propose a three-phase large scale Skyline service selection framework for service composition in clouds. This framework adopts distributed parallel Skyline computation with MapReduce to prune redundant candidate services, and employs parallel multiobjective optimization algorithm based on MapReduce to select Skyline services from the tremendous amount of Skyline services warehouse for composing single service into a set of more powerful Skyline composite services, then applies Top-k query processing technology or multiple attribute decision making support method to select $k$ Skyline composite services from the set of Skyline composite services. Through theoretical analysis, the framework can efficiently solve the service selection problem with large scale services, high-dimensional QoS in cloud computing environment, and quickly generate better composite services with the global optimal QoS.
\end{abstract}

Keywords: Service Selection, MapReduce, Skyline, multi-objective optimization algorithm, Top- $k$, cloud computing

\section{Introduction}

With the development of service/cloud computing and the advent of the era of the Big Data, a tremendous amount of available services which provide the same or similar functionality but differ in QoS attributes (e.g. response time, cost, reputation, availability, reliability, accessibility, trust, security) are deployed on the Internet. However, because of the fast increasing number of services and multiple QoS attributes to be considered, these competing services pose a new challenge to the large scale service selection in service composition: selecting proper service providers that achieve a composition with the best user desired QoS, and efficiently selecting from these huge numbers of web services also brings challenges to the existing approaches. There are a considerable amount of service selection approaches that have been proposed by different researchers and communities.

Approaches of service selection based on QoS currently focus on three directions as follows:

Wenlang Luo is the corresponding author. 
(1) The approaches of service selection based on single objective optimization algorithms. Some single objective algorithms such as genetic algorithm and particle swarm optimization algorithm are employed for service selection based on QoS, which weight each dimensional QoS attribute to combine multiple objectives of QoS to a single. Unfortunately, users would find it hard to accurately weight each dimensional QoS attribute because of lack of priori knowledge, and merged single object makes each dimensional QoS attribute's value invisible to users so that it does not meet their requirements of each attribute. This may lead to the loss of user desired service, thus it is difficult to obtain the optimal composite service with best comprehensive evaluation of global QoS attribute which is provided by users with a high satisfaction. These service selection approaches only obtain single objective optimal solution without substantially solving the problem of service selection with multiple dimensional QoS attributes, for example, simultaneously optimizing response time and cost of composite service for service selection. Hence, this kind of approach has limited the practicability of service selection.

(2) The approaches of service selection based on multi-objective optimization algorithms. Some multi-objective optimization algorithms such as multi-objective genetic algorithm and multi-objective particle swarm algorithm are used for service selection based on QoS, which devise objective function to each QoS attribute instead of weighting them and simultaneously optimizing multiple objective functions to obtain a group of Pareto optimization solutions. Users can select a satisfied solution by their QoS preferences. These service selection approaches have the adaptability to some extent to better meet users' preferences and their personalized requirements.

Two kinds of service selection approaches described in (1) and (2) are both selected from all services of the services warehouse and the selection efficiency is greatly affected by the number of service class and the number of candidate services and the number of its QoS dimensions. These approaches cannot meet real-time demand and assure the quality of those optimal solutions for the problem of service selection with massive services and its high dimensional QoS attributes.

(3) The approaches of services selection based on Skyline computation. To put it simply, the approaches of service selection based on Skyline computation select many skyline services from each service class, and the selected skyline services comprise those services that are not dominated by any other service in the same service class in terms of all QoS attributes. Skyline services are firstly obtained by classic Skyline computation in this approach, then mixed integer programming (MIP) or linear programming (LP) is adopted to select for service composition from these Skyline services. Skyline computation is very time-consuming in face of tremendous amount of services with high dimensional QoS attributes and the obtained Skyline services warehouse has a huge and uncontrollable size. LP approach is very effective when the scale of the problem is small. However, the approach suffers from poor scalability accounting for the exponential time complexity of the applied select algorithms. In MIP or LP, multiple dimensional QoS attributes are weighted to be merged into one objective function. However, it is difficult for users to accurately quantify weight, and when the dimensions of QoS attributes are higher, simultaneously optimizing multiple QoS attributes have low practicability, and only one optimal solution satisfying constraints can be obtained and users have no choice.

The effectiveness of solutions is paid attention to but the efficiency of solutions is ignored in the existing service selection approaches based on single objective or multiobjective optimization algorithm. How to assure high efficiency while improving effectiveness of solutions in a circumstance of tremendous web services? The service selection with massive services and high dimensional QoS causes a great deal of Skyline computation which is beyond the capabilities of existing one-machine solutions and possibly makes memory leak. 
How to select Skyline services rapidly and to effectively produce a good composite service from tremendous amount of web services? Some service selection approaches of parallel Skyline based on MapReduce framework have been proposed for higher efficiency of Skyline service selection, but these approaches only filter out non-Skyline services and don't involve how to select services for service composition.

Currently, it is assumed that all web services are stored in the same platform for most of the existing web service selection approaches based on QoS without the consideration of the distributed environment of large scale services. In cloud computing environment, more and more web services usually are geographically dispersed and massively distributed in different servers that are connected with the Internet. So how to efficiently select services which satisfy different user's personalized and diversified needs from those massive and distributed services becomes an urgent problem to be solved, which is beyond the capability of typical service selection approaches.

In summary, due to the growing number of web services and more and more service's QoS attributes, as well as distributed web service environment in real-world, the traditional service selection approaches are confronted with unprecedented challenges in terms of efficiency and good performance of QoS attributes. Luo et al. [1] pointed out that the era of big data brings new challenges to service selection. The data loads in big data services are typically very large, which brings a big challenge to classical service selection approaches. When calling these big data services in a composite service, there are huge data should be transmitted in the network. However, the model has the exponential time complexity, which performs poorly with high scalability.

In this paper, we proposed a novel service selection framework which is a three-phase large scale Skyline service selection framework for service composition in clouds to meet the challenges and overcome the drawbacks of various approaches. This framework will integrate the advantages of existing service selection approaches and extend it in the MapReduce framework. This framework adopts many key techniques including MapReduce, Skyline computation, multi-objective optimization algorithm, top- $k$ query or multiple attribute decision making and so on, in order to efficiently solve the problem of service selection with massive services and high-dimensional QoS attributes, and to quickly obtain combinational solution of global superior QoS.

\section{A Three-Phase Large Scale Skyline Service Selection Framework}

\subsection{Framework Design}

The three-phase large scale Skyline service selection framework integrates three kinds of approaches as follows:

(1) A distributed parallel skyline massive services screening approach based on MapReduce; (2) A skyline service selection approach of parallel multi-objective optimization algorithm based on MapReduce; (3) A skyline composite service selection approach based on top- $k$ query technique or multiple attribute decision making approach.

As showed in Figure 1, this framework finishes service selection for service composition through the three phases, and the obtained $K(K>=1)$ optimal Skyline composite services are recommended ultimately to be chosen for users. 


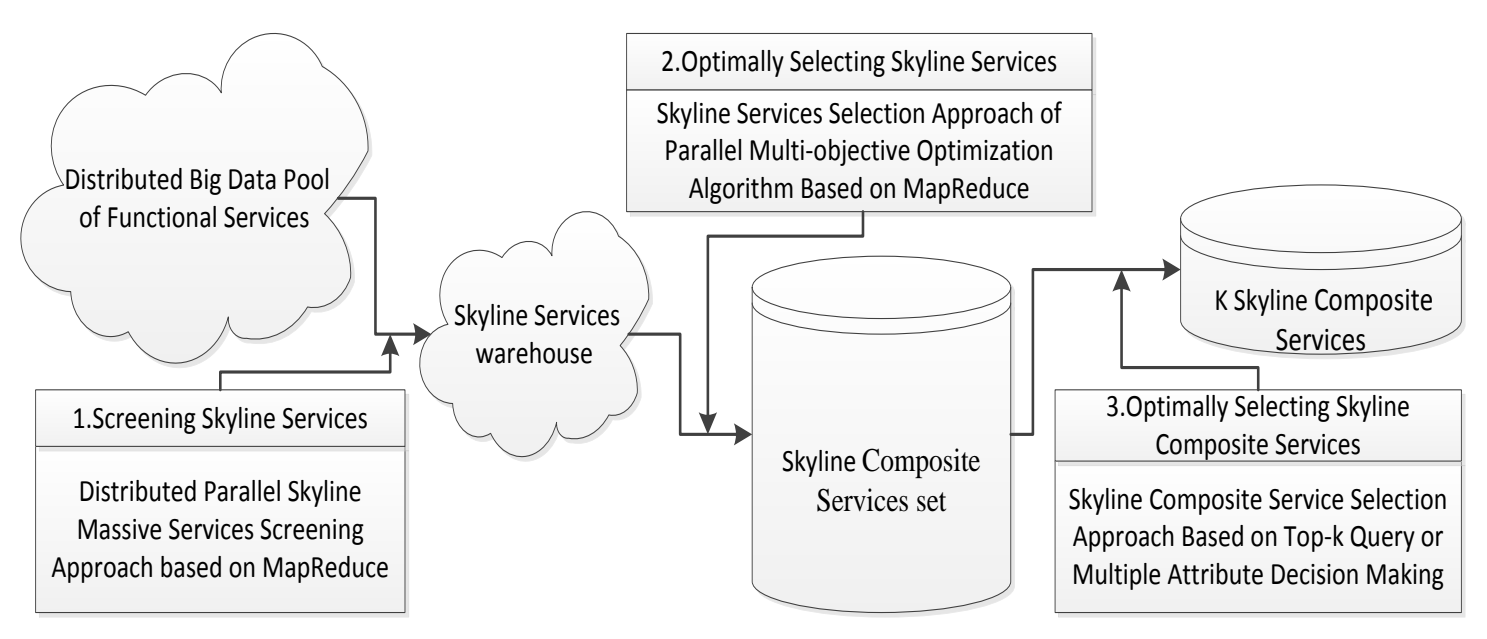

Figure 1. A Three-Phase Large Scale Skyline Service Selection Framework

Phase 1: Screening Skyline services to produce a Skyline services warehouse. First, set the local constraint value of each QoS in each functional service class and select services satisfying these constraint values from the services distributed in all servers. Then, employ the technology of Skyline computation to devise the distributed parallel skyline massive services screening approach based on MapReduce to produce a Skyline services warehouse according to the problem of service selection, and devise real-time updating Skyline algorithm to dynamically maintain the Skyline services warehouse.

The local constraint value of each dimensional QoS attribute of each functional service class is set by users' preferences of individual service class in this phase. If users do not restrict each functional service class, the local constraint check would not be made. To compute Skyline services, existing Skyline computation algorithms can be adopted. These algorithms include block-nested-loops, sort-filter-skyline, divide-and-conquer, bitmap algorithm, index algorithm, nearest-neighbor and so on. Certainly, it is also possible to design a new Skyline computation algorithm. For real-time updating Skyline algorithm, we need to devise it according to the distributed parallel Skyline algorithm.

Phase 2: Optimally selecting Skyline services to form a set of Skyline composite services. Existing multi-objective optimization algorithms which are serial and can be changed into parallel ones based on MapReduce. We can devise the skyline service selection approach of parallel multi-objective optimization algorithm which integrates into the global QoS constraints of composite service according to the problem of service selection. Taking advantage of these algorithms to select Skyline service from the Skyline services warehouse at phase 1 and finally obtain a group of Pareto optimal solutions which are Skyline composite services satisfying global QoS constraints.

The global constraint value of each dimensional QoS attribute of composite service is set by users' preferences for composite service in this phase. If users do not restrict the global QoS of composite service, the global constraint check would not be considered in the process of the design of the skyline service selection approach of parallel multiobjective optimization algorithm based on MapReduce. Existing multi-objective optimization algorithms can be adopted including multi-objective genetic algorithm, multi-objective particle swarm optimization algorithm, multi-objective ant colony optimization algorithm, multi-objective simulated annealing algorithm, non-dominated sorting genetic algorithm and so on. Certainly, it is also possible to design a new multiobjective optimization algorithm, such as multi-objective cuckoo search algorithm.

Phase 3: Optimally selecting Skyline composite services to recommend $K(K>=1)$ optimal Skyline composite services. We design the skyline composite service selection 
approach based on top- $k$ query technique or multiple attribute decision making approach to select top $K$ optimal Skyline composite services which satisfy user's requirements or to select one which has highest score in the set of Skyline composite services from Phase 2.

In this phase, some multiple attribute decision making approaches can be adopted including Simple additive weighting (SAW), Technique for Order Preference by Similarity to an Ideal Solution (TOPSIS), ELECTRE, PROMETHEE and so on; Some top- $k$ query technologies can be adopted including U-Top $K$, U- $k$ Ranks, PT- $k$, GlobalTopK, Expected Rank, E-Score Rank, C-typical-TopK, TA, Stream-Combine, MPro and so on.

\subsection{The Theoretical Analysis of this Framework}

This framework is a container for the kind of massive services selection approaches based on MapReduce in cloud environment. We can combine MapReduce, Skyline computation, multi-objective optimization algorithms and top- $k$ query technique or multiple attribute decision making approach to devise different service selection approaches of massive Skyline services based on MapReduce to efficiently solve service selection problems with massive and distributed services and high-dimensional QoS and quickly obtain Skyline composite services of global optimal QoS. Its advantages are showed as follows:

(1) A class of massive services screening approaches based on distributed parallel Skyline computation algorithms can be adopted in this framework. Different skyline computation algorithms can be parallelized based on MapReduce in the massive services screening approaches to efficiently filter out some non-Skyline services and dynamically maintain Skyline services. This class of approaches can quickly and efficiently handle the screening of massive services in real-time. Some services which don't satisfy QoS constraints or whose QoS are inferior are rejected and the remaining services are accepted. It reduces the number of candidate services for the Skyline service selection of next phase. In the context of massive and distributed services and high-dimensional QoS, it has more rapid response than existing traditional approaches and can meet real-time demand.

(2) A class of Skyline service selection approaches based on parallel multiobjective optimization algorithms can be adopted in this framework. Different multiobjective optimization algorithms can be parallelized based on MapReduce in the Skyline service selection approaches to quickly and efficiently select Skyline service for composite service from the Skyline services warehouse, and finally produce a set of Pareto optimal solutions. Most of multi-objective optimization algorithms are serial, but they have parallel potential, so it is a considerable approach of changing these serial algorithms into parallel ones based on MapReduce to reduce the running time. The achieved Skyline services warehouse in phase 1 will be candidate services of the Skyline service selection approaches, it greatly reduces the space of service selection for service composition, and the obtained solutions are all Skyline composite services, so the class of approaches can improve efficiency of service selection and quality of solutions. This class of approaches can more efficiently and effectively solve the problem of service selection with massive services and high-dimensional QoS attributes compared to the existing service selection approaches based on multi-objective optimization algorithm and based on Skyline computation.

(3) A class of skyline composite service selection approaches based on top- $k$ query technique or multiple attribute decision making approach can be adopted in this framework. Different technologies of top- $k$ query can be used to select top $k$ Skyline composite services satisfying users' requirements from the obtained set of Skyline composite services in the phase 2.

According to the specific application of the service selection problem, different top- $k$ query technologies or multiple attribute decision making methods can be used to obtain 
top $k$ Skyline composite services which have higher scores in all Skyline composite services by the way of setting weight for each dimensional QoS attribute. If the size of the Skyline composite service set is also very large, a considerable solution is to change these technologies of top- $k$ query and multiple attribute decision making methods which are serial into parallel ones based on MapReduce, and the skyline composite service selection approaches are devised based on parallel top- $k$ query or parallel multiple attribute decision making to efficiently select the most satisfying Skyline services for users.

It can be concluded that this framework has strong flexibility and scalability. Three classes of approaches can be combined in this framework to solve the service selection problem with massive services and high-dimensional QoS attributes for service composition in clouds. By this framework, it is possible to more efficiently and effectively obtain Pareto optimal solutions that are Skyline composite services with most optimal QoS satisfying local and global constraints.

\section{Related Work}

A number of service selection approaches have been developed that depend on integer programming approaches or optimization algorithms. Zeng et al. [2] focused on dynamic and quality-driven selection of services, and used LP techniques to find the optimal selection of component services for the composition. Alrifai et al. [3] proposed a hybrid approach that a distributed local selection strategy is integrated with MIP to further improve the optimization performance. But they do not take possible failure in finding a feasible solution into consideration. Alrifai et al. [4] extended and complemented their previous work published in [3], including the QoS computation of complex composite services, by presenting an improved approach for the local selection of quality levels, and so on. Wang et al. [5] proposed an efficient and effective QoS-aware service selection approach, which first employed cloud model to compute the QoS uncertainty for pruning redundant services while extracting reliable services, then, used mixed integer programming to select optimal services. Yu et al. [6] studied the problem of service selection with multiple QoS constraints, they proposed two models for the QoS-based service composition problem: a combinatorial model and a graph model, and a heuristic algorithm was introduced for each model. The proposed heuristic algorithm to find a nearto-optimal solution more efficiently than exact solutions. Despite the significant improvement of this algorithm compared to exact solutions, it does not scale with respect to an increasing number of web services and remains out of the real-time requirements. Claro et al. [7] employed multi-objective optimization techniques to find a set of optimal Pareto solutions from which a user could select the most satisfied web service. Fan et al. [8] presented a new cooperative evolution algorithm consists of stochastic particle swarm optimization and simulated annealing to solve the Web service selection problem.

These LP approaches or optimization algorithms are very effective as the scale of the problem is not very large. However, their scalability is very poor due to the exponential time complexity of the applied search algorithms with the increasing size of the problem because they are very computationally expensive. So they are not suitable for selection oriented to large scale services, as the efficiency is drastically limited by the number of services.

Another possible solution to tackle service selection is to use the Skyline computation which has also received considerable attention recently. Alrifai et al. [9] proposed an approach based on the notion of skyline as a pre-process to select services for composition. This approach reduced the number of candidate services to be considered and improved the efficiency of QoS-based web service select for composition, but this paper only considered the problem of skyline service under the static environment, the efficiency of the proposed algorithm is relatively low, and can't meet the computation of large scale skyline services. Wu et al. [10] introduced the skyline approaches to improve 
the efficiency of selection by using the dominance relationship of skyline to prune services. Yu et al. [11] developed a p-R-tree indexing structure and a dual-pruning scheme to efficiently compute the p-dominant skyline services in the service selection process. Yu et.al [12] developed three skyline algorithms, One Pass algorithm, Dual Progressive algorithm, and Bottom-Up computation framework, to select a set of "best" possible composite services. Yu et al. [13] proposed a multi-attribute optimization approach to tackle the problem of service selection of multiple quality criteria, and developed the Baseline algorithm that adopted a two-level pruning scheme and the operation graph based indexing algorithm which was built upon a novel indexing structure to efficiently compute the skyline service. Benouaret et al. [14] proposed an efficient and flexible Web service selection framework based on QoS, which leveraged two skyline variants-alternative to the traditional skyline.

The main purpose of using Skyline computation for service selection is to filter out non-Skyline service, then to reduce the number of candidate services and to improve the efficiency and effectiveness of service selection for composite service selection in the later phase. But with the increment of web services, its classes and QoS attributes to be considered, the process of skyline service selection is also time consuming, as the skyline query suffers the curse of dimensionality. As the number of services and classes increases, the executing time of computing a service skyline over the large scale services increases in an exponential manner.

Another interesting direction is to develop parallel skyline algorithms that can efficiently compute the service Skyline over a huge numbers of services. Pan et al. [15] made use of skyline with MapReduce to select the appropriate services efficiently and equally for web services selection. They [16] also designed a system architecture and Preprune-Refine framework inspired by MapReduce and skyline techniques for efficient selection from large scale distributed web services. Chen et al. [17] presented a parallel skyline service selection approach to improve the efficiency by employing the MapReduce framework, an angle-based dataspace partitioning approach was employed in their MapReduce based skyline service selection. Wu et al. [18] extended their preliminary work [17], presented the DOPOLO strategy to improve the efficiency of Reduce process, and proposed the First Get First Post algorithm and the MaxDominance algorithm based on different filtering service selection approaches.

These approaches described above mainly discussed how to efficiently screen skyline service from each service class, and not discussed how to select skyline service for service composition from each service class to form composite services with better QoS. But the three-phase large scale Skyline service selection framework for service composition in cloud environment we proposed also includes the approach of selecting skyline services to form composite service for service composition from skyline services warehouse and the approach of selecting composite services desired by users from the generated set of skyline composite services. The framework can provide the ultimate skyline composite services with global optimum QoS to the user.

Several other approaches have been proposed to solve the QoS-aware service selection problem, known to be NP-hard. El Hadad et al. [19] proposed a Web service selection algorithm that satisfied user's preferences, expressed as weights over QoS criteria and as risk levels defining semantically the transactional requirements. Yu [20] presented a relational clustering based collaborative filtering model to predict the QoS of a priori unknown service providers and thus facilitates service users in selecting services that best match their QoS requirements. Ghezzi et al. [21] defined, discussed, and compared three different service selection strategies (local, collaborative, and proxy-based strategies) shedding light on their effectiveness and efficiency. For the service selection scenarios of large scale and distributed services and high dimensional QoS, these approaches have low efficiency, even some approaches may be inefficient while our proposed framework can easily deal with such scenarios. 


\section{Conclusions}

Cloud computing has five essential characteristics including rapid elasticity, resource pooling, on-demand self-service, measured service and broad network access; services in cloud environment have some characteristics including massive, diversity, dynamic variability, scalability, openness, complexity and so on. All these characteristics above provide us with both new opportunities and challenges of solving the problem of large scale services selection in cloud environment. Existing service selection approaches based on distributed and parallel computation are rare. In this paper, we proposed a three-phase large scale Skyline service selection framework for service composition in clouds to efficiently and effectively solve the service selection problem with massive services and high dimensional QoS. The three-phase large scale Skyline service selection framework based on MapReduce can be a considerable approach for solving composite service selection in cloud environment. It can broaden the application field of parallel multiobjective optimization algorithm and provide a reference for future researchers.

Certainly, due to the algorithms, approaches or technologies used in each phase are different in our proposed framework, its performance is also different. How to choose a Skyline computation algorithm and a multi-objective optimization algorithm and a top- $k$ query technology or a multiple attribute decision making approach? And how to combine those approaches of each phase in a reasonable manner to improve the efficiency and effectiveness of service selection? It needs analysis in theory and verification by experiments, which is our next work in the future.

\section{Acknowledgements}

This work is supported in part by the natural science foundation of china (Grant No. 61163062), the Science and Technology Plan Project of Jiangxi Provincial Education Department (Grant No. GJJ14561), the key technology R\&D program of Jiangxi province (Grant No. 20122BBG70161), the Science \& Technology Transformation Program for Universities in Jiangxi Province (Grant No. KJLD13068), the Jiangxi province natural science foundation (Grant No. 20122BAB201038).

\section{References}

[1] Z. L. Luo, Y. Li, and J. W. Yin, "Location: A Feature for Service Selection in the Era of Big Data," Proceedings of the 2013 IEEE 20th International Conference on Web Services, (2013), Santa Clara, pp. 515-522.

[2] L. Z. Zeng, B. Benatallah, A.H.H. Ngu, M. Dumas, J. Kalagnanam, and H. Chang, "Quality driven web services composition", Proceedings of the 12th international conference on World Wide Web, (2003), Budapest, Hungary, pp. 411-421.

[3] M. Alrifai, and T. Risse, "Combining global optimization with local selection for efficient QoS-aware service composition," Proceedings of the 18th international conference on World wide web. (2009), Madrid, Spain, pp. 881-890.

[4] M. Alrifai, T. Risse, and W. Nejdl, "A hybrid approach for efficient Web service composition with endto-end QoS constraints," ACM Transactions on the Web (TWEB), vol. 6, no. 2, Article No. 7, (2012).

[5] S. G. Wang, Z. B. Zheng, Q. B. Sun, H. Zou, and F. C. Yang, "Cloud model for service selection," Proceedings of the IEEE INFOCOM 2011 Workshop on Cloud Computing, (2011), Shanghai, pp. 666-671.

[6] T. Yu, Y. Zhang, and K. J. Lin, "Efficient algorithms for Web services selection with end-to-end QoS constraints", ACM Transactions on the Web (TWEB), vol. 1, no. 1, Article No. 6, (2007).

[7] D. B. Claro, P. Albers, and J. K. Hao, "Selecting web services for optimal composition," Proceedings of the 2nd International Workshop on Semantic and Dynamic Web Processes, (2005), Orlando, Florida, pp. 32-45.

[8] X. Q. Fan, X. W. Fang, and C. J. Jiang, "Research on Web service selection based on cooperative evolution," Expert Systems with Applications, vol. 38, no. 8, pp. 9736-9743, (2011).

[9] M. Alrifai, D. Skoutas, and T. Risse. "Selecting skyline services for QoS-based web service composition," Proceedings of the 19th international conference on World wide web, (2010), Raleigh, North Carolina, pp.11-20.

[10] J. WU, L. CHEN, S. G. DENG, Y. LI, and L. KUANG, "QoS-Skyline Based Dynamic Service Selection”, Chinese Journal of Computers, vol.33, no. 11, pp.2136-2146, (2010).

[11] Q. Yu, and A. Bouguettaya, "Computing service skyline from uncertain qows," IEEE Transactions on 
Services Computing, vol. 3, no.1, pp.16-29, (2010).

[12] Q. Yu, and A. Bouguettaya, "Efficient service skyline computation for composite service selection," IEEE Transactions on Knowledge and Data Engineering, vol. 25, no. 4, pp. 776-789, (2013).

[13] Q. Yu, and A. Bouguettaya, "Multi-attribute optimization in service selection," World Wide Web, vol. 15, no. 1, pp. 1-31, (2012).

[14] K. Benouaret, D. Benslimane, and A. Hadjali. "WS-Sky: An Efficient and Flexible Framework for QoSAware Web Service Selection," Proceedings of the 2012 IEEE Ninth International Conference on Services Computing, (2012), Honolulu, HI, pp. 146-153.

[15] L. M. Pan, L. Chen, and J. Wu, "Skyline web service selection with MapReduce," Proceedings of the 2011 International Conference on Computer Science and Service System (CSSS), (2011), Nanjing, pp739-743.

[16] L. M. Pan, L. Chen, J. Hui, and J. Wu, "QoS-Based Distributed Service Selection in Large-Scale Web Services," Proceedings of the 2011 IEEE International Conference on Services Computing, (2011), Washington, DC, pp.725-726.

[17] L. Chen, L. Kuang, and J. Wu, "MapReduce Based Skyline Services Selection for QoS-aware Composition," Proceedings of the 2012 IEEE 26th International Parallel and Distributed Processing Symposium Workshops \& PhD Forum, (2012), Shanghai, pp. 2035-2042.

[18] J. Wu, L. Chen, Q. Yu, L. Kuang, Y. L. Wang, and Z. H. Wu, "Selecting skyline services for QoS-aware composition by upgrading MapReduce paradigm," Cluster computing, vol. 16, no. 4, pp. 693-706, (2013).

[19] J. El Hadad, M. Manouvrier, and M. Rukoz, "TQoS: Transactional and QoS-aware selection algorithm for automatic Web service composition”, IEEE Transactions on Services Computing, vol. 3, no. 1, pp. 73-85, (2010).

[20] Q. Yu, "QoS-aware service selection via collaborative QoS evaluation”, World Wide Web, vol. 17, no. 1, pp. 33-57, (2014).

[21] C. Ghezzi, V. P. L. Manna, A. Motta, and G. Tamburrelli, "Performance-driven dynamic service selection," Concurrency and Computation: Practice and Experience, Available: http://dx.doi.org/10.1002/cpe.3259, in press, (2014).

\section{Authors}

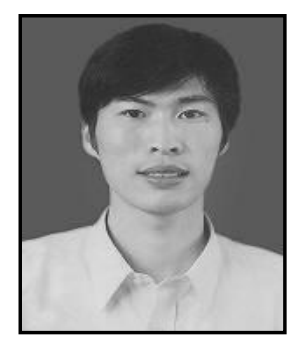

Jinzhong LI is a PhD student at the department of computer science and technology in Tongji University currently, and he has been an associate professor at Jinggangshan University of China. His research interests include services computing, cloud computing, intelligent optimization and evolutionary algorithm. In these fields, he has authored or co-authored over 20 scientific journal paper.

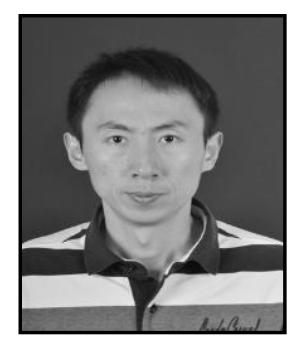

Jintao Zeng is a $\mathrm{PhD}$ student at the College of Surveying and Geo-informatics in Tongji University of China, and he has been an associate professor of the School of Electronic and Information Technology in Jinggangshan University of China. His research interests are services computing, cloud computing, and processing and application of LIDAR data.

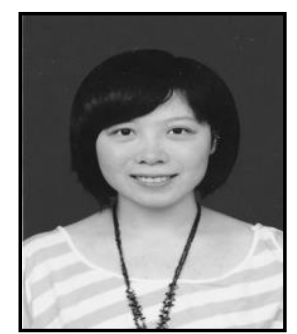

Lei PENG received her MITM degree from the Faculty of Informatics, University of Wollongong, Australia in 2005. Her research interests include Software Engineering Information Technology, WSNs and Cloud Computing. 


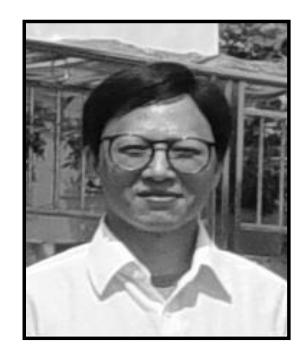

Wenlang Luo (corresponding author) is a professor of the school of electronic and information engineering in Jinggangshan university of China. He received the Ph.D. in physics from Sichuan university of China in 2009. His research interests include computational physics and high performance computing. 